\title{
NOVO SISTEMA DE MONITORIZAÇÃO EEG AMBULATORIAL METODOLOGIA DE ANÁLISE DO REGISTRO E CLASSIFICAÇÃO DAS DESCARGAS EPILÉPTICAS EM 100 PACIENTES
}

\author{
HARLEY P. MUNDIM*, JOSÉ O. CARDEAL **, CARLOS J. R. CAMPOS***
}

\begin{abstract}
RESUMO - Desde 1994 vem sendo utilizado no Brasil um equipamento de monitorização eletrencefalográfica ambulatorial (Holter Cerebral) que foi desenvolvido com união da iniciativa privada e a Universidade Federal de São Paulo - UNIFESP. O objetivo deste estudo foi estabelecer um método de análise, uma classificação das descargas epilépticas encontradas e analisar os resultados da monitorização ambulatorial dos primeiros 100 exames consecutivos de pacientes epilépticos e não-epilépticos, adultos ou crianças, realizados no Setor de Investigação e Tratamento das Epilepsias da UNIFESP. Neste estudo foi possível a identificação de descargas paroxísticas epilépticas e de anormalidades da atividade elétrica cardíaca durante atividades da vida diária dos pacientes.
\end{abstract}

PALAVRAS-CHAVE: monitorização eletrofisiológica ambulatorial, Holter cerebral, desgargas epilépticas.

\section{New system for ambulatory EEG monitoring: analysis and classification of epileptic discharges in 100 patients}

ABSTRACT - We present the first results of a new medical instrument for ambulatory EEG monitoring (Cerebral Holter ) used in Brazil since 1994. One hundred high quality recordings from epileptic and non-epileptic patients allowed the development of a systematic analysis of EEG and ECG signals, and classification of epileptic discharges during daily life activities.

KEY WORDS: ambulatory EEG monitoring; cerebral Holter; epileptic discharges.

A monitorização eletrencefalográfica ambulatorial tem sido um método bastante útil para o diagnóstico das epilepsias e particularmente para aqueles pacientes cujos sintomas fazem diagnóstico diferencial com epilepsia ${ }^{1,2}$. Uma das grandes vantagens desse método em relação ao eletrencefalograma (EEG) convencional é a maior probabilidade de registro de crises epilépticas. O paciente no seu ambiente domiciliar está habitualmente em contato direto com eventuais fatores desencadeantes de suas crises, o que muitas vezes não acontece quando se encontra em ambiente hospitalar. Assim, a monitorização fora do ambiente hospitalar, por não interferir com estes fatores desencadeantes, apresenta uma vantagem importante sobre a monitorização intensiva hospitalar.

A monitorização eletrencefalográfica ambulatorial tornou-se viável somente a partir do desenvolvimento de sistema de registro contínuo da atividade elétrica cardíaca utilizando gravadores de fita cassete, com 4 canais, que foi adaptado para eletrencefalografia ${ }^{3}$. Na década de 80 foi introduzido sistema de monitorização EEG ambulatorial contínua com 8 canais, denominado sistema

*Médico Neurologista, Mestre pela Escola Paulista de Medicina (EPM) - Universidade Federal de São Paulo (UNIFESP); **Professor Adjunto-Doutor da Disciplina de Neurologia do Departamento de Neurologia e Neurocirurgia da EPM - UNIFESP. ***Professor Adjunto-Doutor da Disciplina de Neurologia do Departamento de Neurologia e Neurocirurgia da EPM e Chefe do Setor de Investigação e Tratamento das Epilepsias (SITE) UNIFESP. Aceite: 11-janeiro-1999.

Dr. Carlos José dos Reis de Campos - Rua Pedro de Toledo 655 - 04039-030 São Paulo SP - Brasil. E-mail: cjcamp@ibm.net 
Oxford Medilog 9000, baseado em amplificadores de pequeno tamanho, registro em fitas cassete e leitura em estações computadorizadas. Em 1994, foi desenvolvido no Brasil outro sistema de monitorização EEG ambulatorial contínua, utilizando a tecnologia digital eletrônica desde a gravação e armazenamento do sinal até a análise posterior dos dados registrados.

Houve, inicialmente, muitas dúvidas quando os primeiros sistemas de monitorização eletrencefalográfica ambulatorial foram introduzidos, pois para muitos profissionais parecia um passo para trás explorar apenas 3 ou 4 regiões do cérebro quando o EEG convencional já explorava simultaneamente 16 regiões na maioria dos laboratórios. A opinião "quanto mais canais será sempre melhor" foi gradualmente superada quando se viu que poucos canais também podiam ser bastante úteis, se os dados obtidos de EEG pudessem fornecer as informações que se buscava nos pacientes com e sem epilepsia ${ }^{2,4-6}$. A tendência atual é desenvolver os equipamentos de monitorização eletrencefalográfica utilizando a tecnologia digital eletrônica, mas poucos são os estudos científicos publicados utilizando estes novos sistemas.

O objetivo deste estudo é apresentar e interpretar os resultados da monitorização eletrencefalográfica ambulatorial, analisando as descargas paroxísticas eletrencefalográficas e eletrocardiográficas encontradas no exame, além de verificar as crises clínicas com e sem correlação eletroclínica.

\section{MÉTODO}

\section{Casuística}

Foram utilizados os exames de pacientes epilépticos e não epilépticos, adultos ou crianças, investigados no SITE (Setor de Investigação e Tratamento das Epilepsias da Disciplina de Neurologia da Universidade Federal de São Paulo-UNIFESP). As informações clínicas foram obtidas dos prontuários dos pacientes. Foram utilizados os exames iniciais de 100 pacientes realizados no período de junho 1995 a junho 1996 . Exames de pacientes cujas informações clínicas necessárias fossem julgadas insuficientes foram excluídos. Foram utilizados os critérios preconizados pela ILAE ${ }^{7,8}$ para a classificação das crises epilépticas e das epilepsias.

\section{Equipamentos e programas}

O registro dos sinais foi feito em gravador digital Bioware 2008, desenvolvido através da união da inciativa privada e a UNIFESP, que recebeu em 1994 o Prêmio "Paulo Pinto Pupo" da Academia Brasileira de Neurologia. Neste sistema os sinais elétricos são armazenados, após passar por diferentes etapas de processamento em placa analógica e digital, que incluem pré-amplificação, digitalização, atendendo aos critérios de Nyquist ${ }^{9}$ e compressão dos dados em cartão de memória PCMCIA de 20 Mbytes. Os dados foram lidos em estação de análise constituída de um microcomputador padrão PC-IBM. Um drive de leitura do cartão de memória e sua respectiva placa controladora foram adaptados à estação de análise.

O exame iniciou entre 9 e 12 horas e terminou no dia seguinte entre as 8 e 9 horas, compreendendo período de 18 a 21 horas contínuas, com eletrodos em F3, F4, F7, F8, T3, T4, CZ, segundo o sistema internacional 10-20 recomendado pela Federação Internacional de EEG e Neurofisiologia Clínica ${ }^{10}$. A escolha desses canais, com predomínio em posições fronto-temporais, justifica-se pela menor frequência de anormalidades epileptiformes de projeção em regiões posteriores ${ }^{4}$. O oitavo canal foi usado para o eletrocardiograma (ECG). Além desses 8 canais foram fixados 2 eletrodos auriculares de referência comum e o eletrodo terra, na região da glabela. Os eletrodos, feitos de prata foram colocados na cabeça do paciente com pasta eletrolítica e fixados com colódio.

A análise do exame foi realizada diretamente no vídeo do computador utilizando-se um "software"específico, com a seguinte metodologia de forma sequencial:

- análise do registro dos momentos identificados pelo paciente ou pelo acompanhante como crise clínica ou outra sintomatologia em investigação e anotados em diário ou sinalizados pelo botão de eventos, buscando-se verificar a presença ou ausência de descargas eletrencefalográficas sugestivas de anormalidades ou presença de arritmia cardíaca. Os dados do diário foram utilizados para se estabelecer uma possível correlação eletroclínica.

- as telas de vídeo, com eletrencefalograma e eletrocardiograma foram apresentadas de forma ininterrupta, com tempo de exposição de $0,4 \mathrm{~s}$ por tela, e com o registro numa velocidade de página de $1 \mathrm{~cm} / \mathrm{s}$. Na presença ou suspeita de crise eletrográfica as telas foram marcadas para posterior análise. Escolheu-se montagem bipolar (F3-F7; F7-T3; ECG; F4-F8; F8-T4) com os eletrodos esquerdos na metade superior da tela e os direitos na metade inferior e com o ECG no centro. 
- utilizou-se, a seguir, amostra de $20 \%$ do exame, realizada pelo software, para análise a uma velocidade de $1,5 \mathrm{ou} 3 \mathrm{~cm} / \mathrm{s}$, tela-a-tela, para estudar as descargas paroxísticas como descrito a seguir. As telas com elementos sugestivos de anormalidades foram impressas e analisadas também em folha de papel. Quando se encontrava alguma arritmia cardíaca fazia-se posteriormente avaliação completa do exame utilizando-se montagem com apenas o eletrocardiograma.

As descargas paroxísticas foram classificadas quanto à duração, forma de agrupamento e quanto à morfologia. Quanto à duração foram classificadas em: breves, duração menor que 3 segundos; prolongadas, duração maior ou igual a 3 segundos e menor que 10 segundos; crise eletrográfica, duração maior ou igual a 10 segundos. Quanto à forma de agrupamento foram classificadas em: descargas isoladas, descargas que são seguidas, imediatamente após o seu aparecimento, da atividade elétrica cerebral de fundo e não se repetem num período inferior a 6 segundos; descargas agrupadas, descargas que após a sua manifestação e retorno da atividade de fundo são seguidas por nova descarga paroxística em período inferior a 6 segundos. Quanto à morfologia foram classificadas seguindo as normas da Federação Internacional das Sociedades para Eletrencefalografia e Neurofisiologia Clínica ${ }^{11}$ : ondas agudas (sharp); pontas (spikes); polipontas; complexo ponta-onda e ondas lentas.

\section{RESULTADOS}

Foram analisados os registros de exames de 100 pacientes, um exame por paciente, sendo 48 do sexo masculino e 52 do sexo feminino, com idade variando de 2 a 78 anos.

Apesar dos cuidados tomados durante a instalação do equipamento, o primeiro registro não foi usado em 8 casos: quatro por quebra do cabo de um ou mais eletrodos, três por artefatos constantes originados por má fixação de eletrodos e um por não ter preenchido o diário. Estes exames foram posteriormente repetidos em boas condições técnicas e incluídos na casuística.

Eram epilépticos 67 pacientes, 36 dentre eles (53\%) apresentaram descargas paroxísticas no EEG e nenhum apresentou anormalidades no ECG da monitorização ambulatorial. Os pacientes que não tinham diagnóstico de epilepsia (33) não apresentaram descargas paroxísticas no exame, no entanto $8(24 \%)$ apresentaram a sintomatologia que era investigada; 3 destes com alterações no registro durante esses sintomas: 2 no ECG (extra-sístoles ou taquicardia ventricular) e o outro no EEG (alentecimento da atividade de fundo).

A Tabela 1 apresenta a relação entre a morfologia das descargas encontradas no exame de 36 pacientes epilépticos com a duração (breves ou longas) e forma de agrupamento das descargas (isoladas ou agrupadas).

O tipo de descarga predominante nos exames foi de descargas paroxísticas por ondas agudas, breves e isoladas. Ao mesmo tempo, observou-se que todos os tipos de morfologia estudados (ondas agudas, polipontas, complexos ponta-onda e onda lenta) apresentaram-se com duração tanto breve quanto longa e com tipo de manifestação tanto agrupada quanto isolada. Descargas longas do tipo

Tabela 1. Relação entre a morfologia das descargas encontradas no exame de monitorização eletrencefalográfica ambulatorial realizado em 36 pacientes epilépticos com duração e com forma de agrupamento das descargas.

\begin{tabular}{cccccc}
\hline Descargas paroxísticas & Total de exames & Breves & Longas & Isoladas & Agrupadas \\
\cline { 3 - 5 } & & \multicolumn{4}{c}{ Número de pacientes } \\
\hline Ondas agudas & 26 & 26 & 5 & 26 & 17 \\
PP & 7 & 7 & 2 & 7 & 20 \\
CPO & 20 & 20 & 13 & 4 & 1 \\
Onda lenta & 4 & 3 & 2 & 4 \\
\hline
\end{tabular}

CPO, complexos ponta-onda; $\mathrm{PP}$, complexos polipontas. 
Tabela 2 - Tipo de crise epiléptica e sua relação com a presença de descargas paroxísticas durante o exame de monitorização eletrencefalográfica ambulatorial em 67 pacientes epilépticos.

\begin{tabular}{|c|c|c|c|}
\hline Diagnóstico & Número de pacientes & Descargas paroxísticas & Exame normal \\
\hline $\mathrm{PS}+\mathrm{PC}+\mathrm{P} \rightarrow \mathrm{G}$ & 15 & $5(33 \%)$ & 10 \\
\hline $\mathrm{PC}$ & 6 & $3(50 \%)$ & 3 \\
\hline $\mathrm{PC}+\mathrm{P} \rightarrow \mathrm{G}$ & 12 & $8(66 \%)$ & 4 \\
\hline $\mathrm{PS}+\mathrm{PS} \rightarrow \mathrm{G}$ & 7 & $4 \quad(57 \%)$ & 3 \\
\hline PS & 4 & $1(25 \%)$ & 3 \\
\hline AUS + GTC & 3 & $2(66 \%)$ & 1 \\
\hline GTC & 6 & $2(33 \%)$ & 4 \\
\hline AUS & 4 & $3(75 \%)$ & 1 \\
\hline $\mathrm{GTC}+\mathrm{GM}$ & 3 & $2(66 \%)$ & 1 \\
\hline CI & 3 & $2(66 \%)$ & 1 \\
\hline S. de Lennox-Gastaut & 4 & $3(75 \%)$ & 1 \\
\hline Total & 67 & $35(52 \%)$ & 32 \\
\hline
\end{tabular}

AUS, crise generalizada ausência; GM, crise generalizada mioclônica; GTC, crise generalizada tônico-clônica; PS, crise parcial simples; PC, crise parcial complexa; PS $\rightarrow \mathrm{G}$, crise parcial simples com generalização subseqüente; $\mathrm{P} \rightarrow \mathrm{G}$, parcial (simples ou complexa) com generalização; CI, crise convulsiva não classificada.

poliponta foram observadas em 2 pacientes; em 5 pacientes foram observadas descargas longas por ondas agudas, em 13 pacientes descargas longas do tipo complexos ponta-onda e em 1 paciente descarga longa do tipo onda lenta.

A relação entre o tipo de crise epiléptica e a presença de descargas paroxísticas nos 67 pacientes epilépticos é apresentada na Tabela 2.

Dentre os 44 pacientes com crise parcial 21 pacientes $(48 \%)$ apresentaram descargas paroxísticas. Dos 16 pacientes com diagnóstico de crise generalizada 9 pacientes $(56 \%)$ apresentaram descargas paroxísticas. Dos 4 pacientes com síndrome de Lennox apenas um não apresentou descarga epileptiforme durante o exame.

Observamos descargas por ondas agudas com maior frequência nos pacientes com crises do tipo parcial, embora alguns destes pacientes ( 8 casos) apresentaram descargas por complexos pontaonda, assim como polipontas ( 3 casos). Os pacientes com crise do tipo generalizada apresentaram descargas do tipo complexos ponta-onda. Alguns desses pacientes apresentaram descargas por ondas agudas (4 casos) e polipontas (3 casos).

Observamos também que 11 (16\%) dos pacientes epilépticos apresentaram crise clínica com correlação eletrográfica. A duração da crise detectada pelo EEG da monitorização eletrencefalográfica ambulatorial variou de 3 segundos até 30 minutos. As crises apresentaram morfologia por complexos ponta-onda, polipontas e ondas agudas.

Dentre os 11 pacientes com crise clínica e com correlação eletrográfica, 6 tiveram crise parcial. As crises destes pacientes apresentaram no EEG morfologia tanto por ondas agudas quanto polipontas e complexos ponta-onda. Dos 16 pacientes com crise generalizada, 2 apresentaram crise eletroclínica. As descargas paroxísticas ictais nestes pacientes mostraram morfologia de complexo ponta-onda em um paciente com crise de ausência e de complexo ponta-onda e polipontas no outro paciente. 


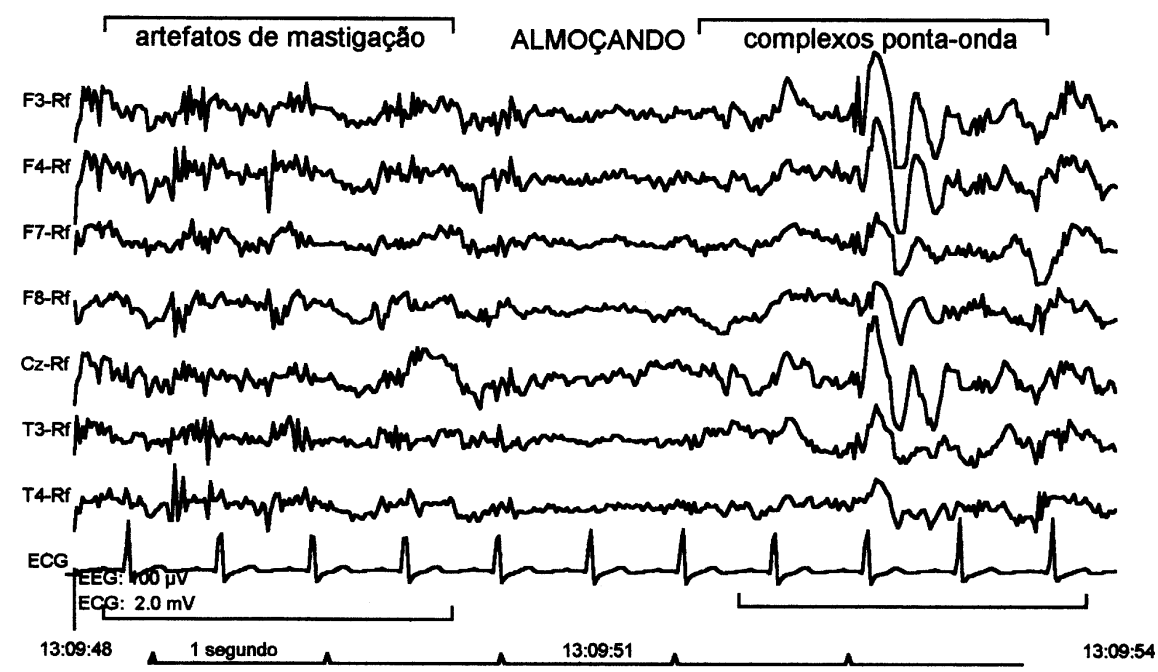

Fig 1. Exemplo de descarga paroxística identificada na presença de artefatos biológicos, com o paciente almoçando.

As crises eletrográficas sem correlação clínica foram manifestadas por $13(19 \%)$ dentre os pacientes epilépticos. Mesmo sem referir nenhuma sintomatologia alguns pacientes apresentaram descargas com duração superior a 10 segundos, variando entre 10 e 38 segundos. Também neste tipo de crise todas as morfologias estiveram presentes: ondas agudas, complexos ponta-onda, polipontas e onda lenta. As descargas foram tanto difusas e bilaterais quanto lateralizadas e focais.

Tanto pacientes com crises parciais quanto pacientes com crises generalizadas apresentaram crise eletrográfica sem correlação clínica. Isto ocorreu em 7 (16\%) dentre os pacientes com crise do tipo parcial e em 4 (25\%) dentre os pacientes com crise generalizada.

Onze pacientes epilépticos apresentavam suspeita de crises pseudoepilépticas associadas. Desses, 5 apresentaram crise clínica sem correlação eletrográfica, 3 deles com diagnóstico de crise parcial complexa relataram ter tido crise "desligamento" ( 2 pacientes) e queda ao solo (1 paciente). Um dos 5 pacientes com crises sem correlação eletrográfica apresentava diagnóstico de síndrome de Lennox-Gastaut, tendo sido relatadas durante o exmae crises caracterizadas como "abalos involuntários". O quinto paciente apresentava diagnóstico de crise generalizada mioclônica e tônicoclônica, e durante o exame referiu crises clínicas caracterizadas como sustos.

Não se verificou dificuldade na diferenciaçãode descargas epilépticas e artefatos, inclusive quando coexistiam no mesmo momento (Fig 1).

Dos 33 pacientes não epilépticos, com idade variando entre 18 e 78 anos, 15 apresentaram diagnóstico de síncope. Destes, 3 (20\%) apresentaram arritmia cardíaca no exame, todos com idade superior a 50 anos. Dentre os 4 pacientes investigados com episódios de tonturas, um com 76 anos, apresentou arritmia cardíaca durante o exame (extrassístoles ventriculares frequentes), 3 apresentaram a tontura durante o exame, sendo que um deles apresentou alentecimento da atividade elétrica cerebral de fundo. Dos 6 pacientes com distúrbios do sono, um deles apresentou episódio de sonambulismo durante o qual o traçado no EEG mostrou ritmos próprios do sono. 


\section{DISCUSSÃO}

Neste estudo, adotamos para o exame de eletrencefalografia e eletrocardiografia ambulatorial o termo monitorização eletrencefalográfica ambulatorial ou Holter Cerebral, sendo o registro feito por período de um dia e uma noite, não se buscando apenas o registro de crises mas também a análise do EEG e do ECG no período intercrítico diurno e noturno.

Conforme recomendação da American Electroencephalographic Society ${ }^{12}$ analisamos as descargas detalhadamente classificando-as quanto a morfologia, duração, forma de agrupamento e topografia. Esta sistematização diagnóstica facilitou a elaboração dos laudos permitindo melhor organizar as informações obtidas a partir do registro eletrofisiológico. A principal indicação da monitorização eletrencefalográfica ambulatorial é para o diagnóstico diferencial das crises clínicas que possam ser de natureza epiléptica. Em nossa casuística, dos 100 exames investigados, 67 eram de pacientes epilépticos e 33 de pacientes que estavam em investigação de distúrbios paroxísticos episódicos. Powell et al. ${ }^{13}$ verificaram que em $75 \%$ dos pacientes que fizeram monitorização eletrencefalográfica e eletrocardiográfica ambulatorial durante 24 horas, buscava-se o esclarecimento diagnóstico de distúrbios súbitos de origem indeterminada.

Dentre os 67 pacientes epilépticos do nosso estudo, 44 apresentavam diagnóstico de crise parcial; 21 desses pacientes apresentaram descargas paroxísticas durante o exame, predominantemente por ondas agudas e, menos frequentemente, por complexos ponta-onda e polipontas. Não se encontrou dificuldade na diferenciação entre descargas epilépticas e artefatos nesses registros.

O simples fato de assistir à televisão, trabalhar com computadores ou passar sob fios elétricos de alta tensão torna o indivíduo susceptível a sinais elétricos de intensidade superiores aos cerebrais captados no escalpo e que podem ser registrados pelo gravador. Este problema, em geral, não existe nos sistemas de eletrencefalografia analógica convencional, pois, nesse caso, o paciente é colocado em ambiente controlado, adequadamente aterrado e, por vezes, até provido de blindagem elétrica. Num sistema de monitorização eletrencefalográfica ambulatorial, estes sinais espúrios devem ser eliminados. Para isto, no equipamento utilizado neste estudo, o sinal de cada canal foi submetido a um filtro de corte (filtro notch) que atenua em 30 vezes o ruído elétrico gerado pela rede $(60 \mathrm{~Hz})$, tornando-os imperceptíveis durante a leitura. Além disso, o problema de geração interna de ruído elétrico por motores de tração que ocorrem nos gravadores com fitas cassete não existem no sistema utilizado neste estudo. Essas características técnicas desse tipo de equipamento permitiram a excelente qualidade dos registros obtidos dos pacientes durante atividades da sua vida diária, tanto em condições de sono como de vigília, como mostrado em trabalhos anteriores ${ }^{14,15}$. Em alguns casos, descargas paroxísticas foram nitidamente diferenciadas de artefatos nos períodos de mastigação, escovação de dentes, conversa ao telefone, durante atividades lúdicas e durante a marcha como mostramos na Figura 1, onde se observa, durante atividade de almoço, a presença de artefatos de mastigação concomitante a descarga paroxística por complexos ponta-onda.

Uma característica importante do equipamento digital utilizado neste estudo foi oferecer a possibilidade de remontagem off-line, após a captação. Com isso, o sinal registrado em um eletrodo foi combinado com sinais de outros eletrodos para montagens bipolares. Esta característica facilitou a diferenciação entre vários tipos de artefatos com as descargas paroxísticas focais ou generalizadas. A utilização de um eletrodo na posição $\mathrm{Cz}$, possibilitou caracterizar com segurança grafoelementos normais do sono e que poderiam ser confundidos com descargas epilépticas.

Em nosso estudo, $16 \%$ dos pacientes epilépticos apresentaram crise clínica com correlação eletrográfica e 7,4\% apresentaram crise clínica sem correlação eletrográfica.

Davidson et al. ${ }^{16}$ encontraram $77 \%$ de crises epilépticas em pacientes que apresentavam crises diariamente e que foram submetidos a monitorização eletrencefalográfica de 24 horas, ao passo que Blumhardt \& Oozeer ${ }^{17}$ encontraram somente $16 \%$ de crises epilépticas em pacientes com frequiência variada de crises. Powell et al. ${ }^{13}$ encontraram crise epiléptica em $50 \%$ dos pacientes que apresentavam 1 crise por semana quando a monitorização eletrencefalográfica ambulatorial foi de 3 dias. 
O registro de crises durante a monitorização eletrencefalográfica ambulatorial parece depender fundamentalmente do tipo de casuística estudada, ou seja, pacientes com sintomas pouco ou muito frequentes, e também da duração do exame, quanto mais prolongado maior a probabilidade do registro de crises. Do mesmo modo, os resultados da documentação de descargas epilépticas interictais é bastante variável na literatura. Na revisão de Ebersole ${ }^{18}$, em torno de 10 a $15 \%$ dos pacientes apresentaram, em casos não selecionados, descargas epilépticas interictais durante o exame.

Em nosso estudo, as descargas epilépticas foram encontradas em $53 \%$ dos pacientes epilépticos. Em 50\% dos pacientes com crise parcial complexa, sem outros tipos de crise parcial, foram observadas descargas epilépticas interictais durante o exame e em $48 \%$ dos pacientes com crise parcial complexa acompanhada de outras crises parciais (parcial simples ou parcial com generalização).

Dentre os 16 pacientes com diagnóstico de crise generalizada, 9 apresentaram descargas epilépticas. As descargas foram, principalmente, por complexos ponta-onda e, mais raramente, por complexos polipontas e, em um paciente, descargas difusas e bilaterais por ondas agudas.

$\mathrm{Chu}^{19}$ utilizou um sistema de monitorização ambulatorial de fitas cassete com 8 canais e montagens bipolares em dois grupos, sendo o primeiro grupo com eletrodos no escalpo e o segundo grupo com eletrodos esfenoidais e também no escalpo, realizando um estudo em pacientes com crise parcial complexa não refratária ao tratamento clínico e em abstinência das medicações no dia do exame. Encontrou 27\% de anormalidades interictais com a monitorização no primeiro grupo e $91 \%$ de anormalidades interictais no segundo grupo. Este estudo é exemplo de utilidade dos equipamentos com 8 canais de captação, qual seja, a possibilidade de estudos com eletrodos zigomáticos ou esfenoidais. Os resultados diferentes entre os estudos têm relação principalmente com a diversidade e tamanho das casuísticas, com a metodologia envolvida na análise dos exames e também com a diferença dos equipamentos empregados em cada estudo. Em nosso estudo, por exemplo, a análise amostral de $20 \%$ das telas de vídeo possibilitou a detecção de descargas interictais que não tinham sido observadas durante a leitura rápida do registro total, quando se buscava identificar, prioritariamente, a presença de crises eletrográficas.

Também em nosso estudo, tanto pacientes com crises parciais quanto pacientes com crises generalizadas apresentaram variados tipos de morfologia, duração e agrupamento das descargas. Assim, 14\% dos pacientes com crises parciais apresentaram descargas paroxísticas por complexo ponta-onda. Complexos polipontas, como única alteração do exame, foram observadas em apenas dois pacientes com crise parcial. Por outro lado, 25\% dos pacientes com diagnóstico de crises generalizadas apresentaram morfologia por ondas agudas localizadas ou generalizadas. Dois desses casos de crise generalizada nos quais as ondas agudas mostraram-se lateralizadas podem na realidade corresponder a crise parcial. Uma das vantagens da monitorização eletrencefalográfica prolongada é detectar o que muitos EEG convencionais repetidos não evidenciaram e, com isso, melhor documentar a descarga epiléptica, possibilitando o diagnóstico correto.

A decisão neste estudo de classificar o tipo de apresentação das descargas epilépticas interictais durante a monitorização ambulatorial teve o intuito de não valorizar somente as crises clínicas referidas pelo paciente ou acompanhantes. Não encontramos estudos que apresentassem uma classificação das descargas epilépticas registradas com este tipo de exame em relação à duração ou ao tipo de agrupamento e, assim, a análise das descargas epilépticas interictais permitiu a sistematização: crises breves e longas; crise eletrográfica; crises isoladas e agrupadas.

Observou-se que o tipo de descarga predominante nos exames com relação à duração e ao modo de agrupamento das descargas paroxísticas foram breve e isolada, respectivamente. Todos os tipos de grafoelementos patológicos estudados apresentaram-se com duração tanto breve quanto longa e com tipo de manifestação tanto agrupada quanto isolada. A utilidade dessa classificação não pode ser avaliada apenas com este estudo, mas muitos aspectos poderão ser esclarecidos ulteriormente. Por exemplo, é possível que muitas descargas longas subclínicas possam interferir com processos cognitivos do paciente, o que poderá ser elucidado por testes neuropsicológicos adequados. Por outro lado, poderá ser estabelecida alguma correlação entre padrão de agrupamento das descargas e prognóstico da epilepsia. 
As crises convulsivas geralmente não passam desapercebidas nem pelo acompanhante nem pelo paciente e não precisam da monitorização eletrencefalográfica ambulatorial para estabelecer sua freqüência. Já as crises parciais simples ou as crises parciais complexas quando muito freqüentes ou muito sutis, podem se beneficiar do uso da monitorização eletrencefalográfica ambulatorial. Também beneficiam-se desse instrumento diagnóstico pacientes com crises subclínicas de ausência expressas por complexos ponta-onda. Neste tipo de crise, o paciente ou acompanhante não a percebe, tornando impossível ou duvidosa a correta informação sobre o número e duração das crises sem a utilização de algum tipo de monitorização eletrofisiológica.

Foram encontradas descargas paroxísticas com duração de 10 segundos ou mais (crise eletrográfica) em 13 pacientes epilépticos (19\%). Em nenhum desses casos as informações obtidas do diário, descritas pelo paciente ou pelo acompanhante, corresponderam a manifestações compatíveis com crise clínica concomitante a essas descargas.

Alguns aspectos ainda não foram explorados como, por exemplo, a correlação entre tipos de distúrbios clínicos e descargas epilépticas longas, além de outras questões. Há predominância de certos distúrbios de acordo com o tipo de descarga epiléptica encontrada? Descargas por complexos ponta-onda estão mais associadas a distúrbios cognitivos do que descargas por polipontas ou por ondas agudas? A presença ou ausência de sintomas depende da região de predomínio das descargas? Os pacientes com diferentes tipos de descargas são de controle clínico mais difícil? Estão mais envolvidos em acidentes? Quais são os reais riscos e benefícios envolvidos por uso de drogas antiepilépticas com o intuito de "limpar "o EEG?

Neste estudo, a monitorização eletrencefalográfica ambulatorial com o intuito de diagnosticar crise pseudoepiléptica foi utilizada com muita ressalva. Com certeza o EEG prolongado pela monitorização ambulatorial aumenta a probabilidade de registrar crises tanto verdadeiras quanto falsas. Contudo as informações sobre o evento clínico estarão limitadas às descrições obtidas do diário, sendo esta a principal deficiência da monitorização ambulatorial a ser levada em conta na avaliação de pacientes com suspeita de crise pseudoepiléptica.

A monitorização eletrencefalográfica ambulatorial é de grande ajuda quando confirma o diagnóstico de epilepsia ao mostrar que um ataque está realmente associado a descargas paroxísticas tipicamente epilépticas. Várias investigações têm mostrado a sua utilidade em esclarecer a natureza epiléptica de comportamentos que eram considerados previamente como sendo de natureza psiquiátrica ${ }^{20}$.

$\mathrm{Na}$ nossa casuística encontramos 11 pacientes epilépticos com suspeita de crises pseudoepilépticas associadas às crises epilépticas, 5 deles apresentaram a "crise" durante a monitorização; 3 desses pacientes apresentavam diagnóstico de crise parcial complexa com possível crise pseudoepiléptica associada; 2 deles com episódios de "desligamentos" e 1 com episódios de queda súbita ao solo; 1 paciente que apresentava diagnóstico de síndrome de Lennox-Gastaut, além das crises epilépticas apresentava movimentos involuntários anormais; 1 paciente com crise generalizada tônico-clônica associada a crises generalizadas mioclônicas apresentou episódios de "sustos" durante o exame caracterizados por movimentos rápidos sugerindo crises mioclônicas. Todos esses pacientes não apresentaram descargas epilépticas no exame durante as crises referidas.

O diagnóstico pode tornar-se ainda mais difícil quando o ataque em questão é similar a uma crise parcial complexa com alteração apenas do nível de consciência. Na falta de correlação eletroclínica durante a monitorização ambulatorial pode-se inferir o diagnóstico de crise pseudoepiléptica, embora não se descarte a possibilidade de crise parcial sem repercussão em registros usando eletrodos em couro cabeludo. Isto acontece principalmente em algumas crises parciais simples cuja origem do foco encontra-se localizado profundamente ${ }^{21}$.

Assim, os nossos estudos possibilitaram o estabelecimento de uma metodologia de análise do registro de monitorização eletrencefalográfica ambulatorial e uma classificação das descargas 
epilépticas encontradas. A utilização da metodologia de análise e da classificação estabelecidas permitiram a identificação de descargas paroxísticas epilépticas e de anormalidades da atividade cardíaca durante atividades da vida diária dos pacientes. Nos pacientes com diagnóstico final de epilepsia o registro da atividade elétrica cardíaca não trouxe elementos de valor diagnóstico.

\section{REFERÊNCIAS}

1. Ives JR, Woods JF. Four-channel 24-hour cassette recorder for long term EEG monitoring of ambulatory patients. Electroenceph Clin Neurophysiol 1975;39:88-92.

2. Ebersole JS, Bridgers SL. Direct comparison of 3- and 8-channel ambulatory cassette EEG with intensive inpatient monitoring. Neurology 1985;35:846-854.

3. Marson GB, Mckinnon JB. A miniature four-channel cassette recorder for many applications. Control and Instrumentation 1972;4:46-47.

4. Leroy RF, Ebersole JS. An evaluation of ambulatory, cassete EEG monitoring: I. Montage design. Neurology 1983;33:1-7.

5. Ebersole JS, Leroy RF. An evaluation of ambulatory, cassette EEG monitoring. II - Detection of interictal abnormalities. Neurology 1983;33:8-18.

6. Bridgers SL, Ebersole JS. The clinical utility of ambulatory cassette EEG. Neurology 1985;35:166-173.

7. Commission on Classification and Terminology of the International Leagle Against Epilepsy. Proposal for revised clinical and electroencephalographic classification of epileptic seizures. Epilepsia 1981;22:489-501.

8. Commission on Classification and Terminology of the International Leagle Against Epilepsy. Proposal for classification of epilepsies and epileptic syndromes. Epilepsia 1985;26:268-278.

9. Franklin GF, Powell JD. Digital control of dynamic systems. London: Addinson-Wesley, 1980:185-203.

10. Jasper HH. The ten twenty electrode system of the international federation. Electroenceph Clin Neurophysiol 1958;10:371-375.

11. Chátrian GE, Bergamini L, Dondey M, Klass WD, Lennox-Buchthal M, Petersen L. Glossary of terms most commonly used by clinical electroencephalographers. Electroenceph clin Neurophysiol 1974;37:449-460.

12. American Electroencephalographic Society. Guidelines for long-term neurodiagnostic monitoring in epilepsy. J Clin Neurophysiol 1985;2:419-452.

13. Powell TE, Harding GFA, Jeavons PM. Ambulatory EEG Monitoring: a preliminary follow-up study. In Ross E et al. (eds). Epilepsy in young people. London: J. Wiley and Sons, 1987:131-139.

14. Kida J, Park EJ, Campos CJR. Quantificação de artefatos em registros de EEG ambulatorial (Holter cerebral): relato preliminar de 8 casos. Rev Neurociências 1998;6:11-16.

15. Park EJ, Kida J, Cardeal JO. Atenuação de artefatos fisiológicos pela interferência de complexos ponta-onda de $3 \mathrm{~Hz}$ no EEG Ambulatorial: estudo preliminar de 8 casos. Rev Neurociências 1998;6:6-10.

16. Davidson DLW, Fleming AMM, Kettles A. Use of ambulatory EEG monitoring in a neurological service. In: Dam L, Gram L, Penry JK (eds). Advances in epileptology: XIIth International Epilepsy Symposium. New York: Raven Press, 1981:319-321.

17. Blumhardt L, Oozeer R. Simultaneous ambulatory monitoring of the EEG and ECG in patients with unexplained transient disturbances of consciouness. In Stott FD, Wright SL, Raftery EB (eds). ISAM 1981: Proceedings of the Fourth International Symposium on Ambulatory Monitoring. London: Academic Press, 1982:171-182.

18. Ebersole JS. Clinical utility of cassette EEG in adult seizure disorders. In Ebersole JS (ed). Ambulatory EEG Monitoring. New York, Raven Press, 1989:111-127.

19. Chu NS. Long-term ambulatory cassete EEG with sphenoidal recording in complex partial seizures. Epilepsia 1991;32:351-357.

20. Stores G. Psychological aspects of nonconvulsive status epilepticus in children. J Child Psychol Psychiatry 1986;27:575-582.

21. Ebersole JS, Keilson MJ, Wade PB. Clinical usefulness of ambulatory EEG. American Academy of Neurology Annual Meeting Workshop 1993;250:47-67. 